\title{
Decentralized and Distributed Transient Control for Microgrids
}

\author{
Christoph Kammer, Alireza Karimi
}

\begin{abstract}
This paper treats the problem of primary and secondary control design in low-inertia power grids with mixed lines and a large amount of inverter-interfaced generation. A dynamic phasor model is developed that represents the electromagnetic and electromechanic dynamics of lines, inverters, synchronous machines and constant power loads. The model offers a straightforward way to combine white-, grey- and blackbox models, and its structure lends itself well to control design. In a next step, a novel method to design fixed-structure robust controllers based on the frequency response of multivariable systems and convex optimization is presented. The method offers an intuitive way to define the control performance specifications, and is able to directly design discrete-time controllers. Finally, the potential of the control design method and the dynamic phasor model is demonstrated in a comprehensive example. In three scenarios it is illustrated how the approach can be used to significantly improve frequency and voltage transient performance in low-inertia power grids. Decentralized as well as distributed architectures for primary and secondary control are studied, and results are validated in simulation.
\end{abstract}

Index Terms-Microgrid, Power System Transients, Robust Control, Distributed Control

\section{INTRODUCTION}

Ensuring stability and transient performance in low-inertia power grids is a challenging and relevant control problem. The steady increase of renewable generation and inverter-interfaced loads affects power quality and degrades grid stability. Today, the effect is most pronounced in low- and medium-voltage distribution grids and islanded grids. In these grids, lines are typically short, which introduces strong coupling between generation units. As more and more traditional large power plants are shut down, low inertia and variable generation also become critical issues on the transmission level.

In these circumstances, the classical droop control approach exhibits several major flaws. Being only a proportional controller, it is not possible to properly decouple control of active and reactive power, and oscillatory behavior in frequency and voltage magnitude cannot be damped. Furthermore, traditionally secondary control is implemented as a centralized controller with a relatively low bandwidth. Distributed approaches to secondary control are able to significantly decrease the time constant of the integral action and thus improve the performance of the grid.

In the literature, a common control approach for frequency control of voltage source inverters (VSIs) in low-inertia grids

Christoph Kammer and Alireza Karimi are with the Automatic Control Laboratory at EPFL, 1015 Lausanne, Switzerland.

This work was supported by the Swiss Competence Center for Energy Research_Future Swiss Electrical Infrastructure (SCCER_FURIES). is based on the concept of virtual inertia, where VSIs are used to emulate the behaviour of synchronous generators (SGs) [1], [2], [3], [4]. However, the employed controller structures are often simplistic, and the control design is not modelbased. In general, no methodical control design approach on a systemic level is presented, and stability analysis of the grid is performed a posteriori.

A further issue is that the $R / X$-ratio of lines in mediumand low-voltage grids is often close to or below 1 , meaning the standard decoupling assumption between active and reactive power is not applicable. In [5] an approach is presented where the droop control equations are modified via a transformation matrix based on the $R / X$-ratio of the individual lines in order to decouple the controller. A related solution is the concept of virtual impedance control, where a feedforward controller is added to the VSI control scheme in order to emulate a resistive or inductive output impedance [6], [7], [8]. While this approach has the potential to greatly improve control performance and power quality, no systematic approach to the tuning of the feedforward gains is given, and the stability can again only be evaluated a posteriori.

The work in [9], [10] advocates the use of a primary controller with multiple degrees of freedom in order to improve transient stability and performance. However, no method to achieve a desired control performance is presented, and the electromagnetic line dynamics are neglected. In [11], [12] the global stability of droop control is discussed, but the line resistance and electromagnetic dynamics are neglected, and control design is not treated. A systematic control design approach is pursued in [13], where an improved primary controller for a low-inertia grid is designed based on the Block Gerschgorin theorem. The drawback of this approach is that it often yields very conservative results for strongly coupled systems such as the power grid.

Another approach is the design of decentralized and distributed state-space controllers based on a full small-signal model of the grid. In [14], [15], [16], [17] the application of optimal control design techniques for microgrids with multiple VSIs has been explored. Classical $H_{\infty}$ methods are used in [18], [19] to improve primary frequency control, but as the problem is posed in an LFT form, it is difficult to correctly define the desired performance specifications. In [20], [21], a new $H_{\infty}$ control design approach based on convex optimization is used to design low-order fixed-structure voltage controllers that fulfill specific performance specifications and are robust towards parametric uncertainty. A common problem of these state-space control design approaches is that they do not scale well for larger systems. Thus, considering more 
detailed models of generation units or larger grids quickly makes their application infeasible.

\section{A. Contributions}

The articles cited so far generally require a small-signal model of the grid for control design or stability analysis, which becomes exceedingly complex for large systems. To address this issue, in Section II of this paper a dynamic model for low- and medium-voltage distribution grids based on a dynamic phasor approach is presented. The model is suitable for arbitrary topologies and is able to accurately represent the electromagnetic and electromechanic dynamics of VSIinterfaced generation as well as synchronous generators and constant power loads. It is of a significantly reduced complexity compared to a small-signal model, and relies on a modular structure that makes it straightforward to combine white-, grey- and black-box models of various grid components. While similar models have been used for stability analysis for grids with multiple VSIs in [22], [23], [24], and for control design of single inverters in [25], their application to controller synthesis for complete grids with multiple parallel generation units remains largely unexplored.

In Section III a novel method to design fixed-structure robust controllers based on the frequency response of multivariable systems and convex optimization is presented. Distinct advantages of the method are that no parametric model is required for the design, and that it is possible to directly design discrete-time controllers with the desired order and structure. Furthermore, unlike in classical $H_{\infty}$ methods, the control performance can be specified as constraints on the norm of individual weighted sensitivity functions, which allows for an intuitive problem formulation. Lastly, it is straightforward to include multimodel uncertainty in the design, which can be used to guarantee robustness e.g. against topology changes or generator outages.

In Sections IV-B and IV-D the control design method is illustrated in a three-part scenario in an islanded mediumvoltage grid with mixed lines, multiple VSIs and a synchronous generator. First, based on the dynamic phasor model of the grid, a low-order decentralized primary controller is calculated that significantly improves frequency and voltage transient performance as compared to droop control. Then, in a second step the controller is robustified against a change in the grid topology. Finally, in the third part a distributed control layer is added in order to remove the frequency steady-state error occuring after a load change. This secondary controller has a similar bandwidth as the improved primary controller and does not rely on a centralized architecture, thus removing the single point of failure and allowing for a modular structure as compared to classical secondary control.

This paper can be viewed as a combination of two conference papers [26], [27], with the following extensions. The grid model is extended to include synchronous generators, and a new approach for distributed control design is presented. Furthermore, simulation examples with three new scenarios are provided.

\section{DyNAMiC Phasor MOdEL}

In this section a model based on dynamic phasors will be presented that lends itself well towards controller synthesis. The dynamic phasor model is a frequency-domain model that is able to accurately represent the electromagnetic and electromechanic dynamics of the lines as well as various generation units and other grid components. Dynamic phasors have originally been developed for power system stability analysis in large-scale grids [28]. The main advantage of this approach is that a three-phase voltage can be reduced to only two values: the phase angle and the voltage magnitude. The resulting model is significantly simpler than a full smallsignal model, which in general proves to be too unwieldy for controller synthesis.

\section{A. Dynamic Power Flow Equations}

The dynamic formulation of the power flow equations form the core of the model. The balanced, three-phase voltage at bus $i$ can be represented in the phasor notation:

$$
\mathbf{U}_{i}(t) \angle \theta_{i}(t)=\sqrt{2} \mathbf{U}_{i}(t)\left[\begin{array}{c}
\cos \left(\bar{\omega} t+\theta_{i}(t)\right) \\
\cos \left(\bar{\omega} t+\theta_{i}(t)-2 \pi / 3\right) \\
\cos \left(\bar{\omega} t+\theta_{i}(t)+2 \pi / 3\right)
\end{array}\right]
$$

with $\theta_{i}(t), \mathbf{U}_{i}(t)$ being the voltage angle in rad and the lineto-ground RMS voltage magnitude at bus $i$, and $\bar{\omega}$ being the nominal grid frequency in $\mathrm{rad} / \mathrm{s}$.

For low- and medium-voltage distribution grids, lines can be modeled as R-L elements. The dynamic power flow equations in such a line linearized around the nominal frequency and voltage can be written in the following Laplace transfer function form (see [28]):

$$
\begin{aligned}
P_{i j}(s)= & G_{i j}^{\omega \rightarrow P}(s)\left(\omega_{i}(s)-\omega_{j}(s)\right) \\
& +G_{i j}^{U \rightarrow P}(s)\left(U_{i}(s)-U_{j}(s)\right) \\
Q_{i j}(s)= & G_{i j}^{U \rightarrow Q}(s)\left(U_{i}(s)-U_{j}(s)\right) \\
& +G_{i j}^{\omega \rightarrow Q}(s)\left(\omega_{i}(s)-\omega_{j}(s)\right)
\end{aligned}
$$

where $P_{i j}(s), Q_{i j}(s)$ are the Laplace transform of the active and reactive power transmitted from bus $i$ to bus $j, \omega_{i}(s)$ is the Laplace transform of the grid frequency in $\mathrm{rad} / \mathrm{s}$ at bus $i$ and $U_{i}(s)$ is the Laplace transform of the line-to-ground RMS voltage magnitude at bus $i . G_{i j}^{x \rightarrow y}$ are transfer functions between the inputs $x$ and outputs $y$ between buses $i, j$, and are defined as follows:

$$
\begin{aligned}
& G_{i j}^{\omega \rightarrow P}(s)=3 \frac{\bar{\omega} L_{i j}}{\left(L_{i j} s+R_{i j}\right)^{2}+\left(\bar{\omega} L_{i j}\right)^{2}} \bar{U}^{2} \frac{1}{s} \\
& G_{i j}^{U \rightarrow P}(s)=3 \frac{L_{i j} s+R_{i j}}{\left(L_{i j} s+R_{i j}\right)^{2}+\left(\bar{\omega} L_{i j}\right)^{2}} \bar{U} \\
& G_{i j}^{\omega \rightarrow Q}(s)=3 \frac{L_{i j} s+R_{i j}}{\left(L_{i j} s+R_{i j}\right)^{2}+\left(\bar{\omega} L_{i j}\right)^{2}} \bar{U}^{2} \frac{1}{s} \\
& G_{i j}^{U \rightarrow Q}(s)=3 \frac{\bar{\omega} L_{i j}}{\left(L_{i j} s+R_{i j}\right)^{2}+\left(\bar{\omega} L_{i j}\right)^{2}} \bar{U}
\end{aligned}
$$

where, $R_{i j}, L_{i j}$ are resistance and inductance of the line and $\bar{U}$ is the nominal line-to-ground RMS voltage. The argument $(s)$ is omitted in the rest of the paper. 
Assuming $R / X$ is small, it is interesting to note that the steady-state formulation of (2) and (3) reduces to the wellknown static power flow equations:

$$
\begin{aligned}
P_{i j} & =3 \frac{X}{Z^{2}} \bar{U}^{2} \frac{1}{s}\left(\omega_{i}-\omega_{j}\right) \\
Q_{i j} & =3 \frac{X}{Z^{2}} \bar{U}\left(U_{i}-U_{j}\right)
\end{aligned}
$$

\section{B. Line Power Flows Model}

In this subsection the transfer function from the bus frequencies and voltages to the active and reactive line power flows is developed. We assume that every bus in the grid is connected to either a VSI, an SG or a load, and that any zero-injection buses have been eliminated (e.g. using Kron reduction [29]). Then, dividing the buses into VSI buses, SG buses and load buses, we can write:

$$
\left[\begin{array}{c}
P_{\mathcal{I}} \\
Q_{\mathcal{I}} \\
P_{\mathcal{S}} \\
Q_{\mathcal{S}} \\
P_{\mathcal{L}} \\
Q_{\mathcal{L}}
\end{array}\right]=\left[\begin{array}{lll}
G_{1} & G_{2} & G_{3} \\
G_{4} & G_{5} & G_{6} \\
G_{7} & G_{8} & G_{9}
\end{array}\right]\left[\begin{array}{c}
\omega_{\mathcal{I}} \\
U_{\mathcal{I}} \\
\omega_{\mathcal{S}} \\
U_{\mathcal{S}} \\
\omega_{\mathcal{L}} \\
U_{\mathcal{L}}
\end{array}\right]
$$

Let $p$ be the number of VSI buses, $q$ the number of sync. generator buses and $l$ the number of load buses in the grid. $P_{\mathcal{I}}, Q_{\mathcal{I}} \in \mathbb{R}^{p \times 1}, P_{\mathcal{S}}, Q_{\mathcal{S}} \in \mathbb{R}^{q \times 1}, P_{\mathcal{L}}, Q_{\mathcal{L}} \in \mathbb{R}^{l \times 1}$ are vectors with the active and reactive power injected by the VSIs, sync. generators and loads (i.e. load powers usually have a negative sign). The matrix transfer functions $G_{i}(i=1, \ldots, 9)$ are constructed using the power flow transfer functions in equations (2) and (3), where $G_{1}$ is of dimension $2 p \times 2 p$, $G_{2}$ of $2 p \times 2 q, G_{3}$ of $2 p \times 2 l$, with the dimensions of $G_{4, \ldots, 9}$ accordingly.

As example, consider a radial grid with 3 buses, with a load connected at bus 1, a VSI at bus 2 and an SG at bus 3. Then, $G_{1,2,3}$ would be:

$$
\begin{aligned}
G_{1} & =\left[\begin{array}{ll}
G_{21}^{\omega \rightarrow P}+G_{23}^{\omega \rightarrow P} & G_{21}^{U \rightarrow P}+G_{23}^{U \rightarrow P} \\
G_{21}^{\omega \rightarrow Q}+G_{23}^{\omega \rightarrow Q} & G_{21}^{U \rightarrow Q}+G_{23}^{U \rightarrow Q}
\end{array}\right] \\
G_{2} & =\left[\begin{array}{ll}
-G_{23}^{\omega \rightarrow P} & -G_{23}^{U \rightarrow P} \\
-G_{23}^{\omega \rightarrow Q} & -G_{23}^{U \rightarrow Q}
\end{array}\right] \\
G_{3} & =\left[\begin{array}{ll}
-G_{21}^{\omega \rightarrow P} & -G_{21}^{U \rightarrow P} \\
-G_{21}^{\omega \rightarrow Q} & -G_{21}^{U \rightarrow Q}
\end{array}\right]
\end{aligned}
$$

The frequency and voltage at the load buses is generally unknown. Assuming constant power loads, the dynamics are reformulated such that the power drawn by the loads enters the system as a disturbance. Thus, the power injected by the generators can be written as a function of the generator bus phasors, with the load power acting as a disturbance:

$$
\left[\begin{array}{c}
P_{\mathcal{I}} \\
Q_{\mathcal{I}} \\
P_{\mathcal{S}} \\
Q_{\mathcal{S}}
\end{array}\right]=G_{\text {grid }}\left[\begin{array}{c}
\omega_{\mathcal{I}} \\
U_{\mathcal{I}} \\
\omega_{\mathcal{S}} \\
U_{\mathcal{S}}
\end{array}\right]+G_{d}\left[\begin{array}{c}
P_{\mathcal{L}} \\
Q_{\mathcal{L}}
\end{array}\right]
$$

with

$$
\begin{aligned}
G_{\text {grid }} & =\left[\begin{array}{ll}
G_{1}-G_{3} G_{9}^{-1} G_{7} & G_{2}-G_{3} G_{9}^{-1} G_{8} \\
G_{4}-G_{6} G_{9}^{-1} G_{7} & G_{5}-G_{6} G_{9}^{-1} G_{8}
\end{array}\right] \\
G_{d} & =\left[\begin{array}{l}
G_{3} G_{9}^{-1} \\
G_{6} G_{9}^{-1}
\end{array}\right]
\end{aligned}
$$

This formulation assumes $G_{9}$ to be invertible, which is always the case if all buses in the grid are connected.

It is important to note that $G_{\text {grid }}$ describes the dynamics of the lines, but does not contain the dynamics of the generation units and sensors. The following sections will present a way to model these dynamics, and create a complete model of the system.

\section{Voltage Source Inverter Model}

A specific advantage of the presented formulation is that grey- and black-box models of VSIs can directly be incorporated, and no knowledge of the internal control loops and dynamics is required. Assuming a VSI operating in FrequencyVoltage mode, a simple way to model the closed-loop dynamics is as an ideal voltage source with the following first-order dynamics:

$$
\begin{aligned}
{\left[\begin{array}{l}
\omega_{\mathcal{I}} \\
U_{\mathcal{I}}
\end{array}\right] } & =G_{\mathcal{I}}\left[\begin{array}{c}
\bar{\omega}_{\mathcal{I}} \\
\bar{U}_{\mathcal{I}}
\end{array}\right] \\
G_{\mathcal{I}} & =\operatorname{diag}\left(\frac{1}{\tau_{\omega} s+1}, \frac{1}{\tau_{U} s+1}\right)
\end{aligned}
$$

where $\bar{\omega}_{\mathcal{I}}, \bar{U}_{\mathcal{I}}$ are the desired VSI bus frequency and voltage magnitude, and $\tau_{\omega}, \tau_{U}$ are the closed-loop time constants of the frequency and voltage control loop. If the VSI is outfitted with an $L$-type output filter, a simple way to model it is to lump it with the parameters of the lines connected to the VSI.

The dynamics of the VSI transfer function can easily be extended to include more complicated output filters, resonance modes and time delays. If the internal control loops and parameters are known, the model can also be augmented to include an exact formulation of the complete VSI dynamics.

\section{Synchronous Generator Model}

The main frequency dynamics of a synchronous generator are well represented through the swing equation [30]:

$$
\frac{2 H}{\bar{\omega}} \dot{\omega}_{\mathcal{S}}=G_{\mathrm{PM}} \bar{P}_{\mathcal{S}, m}-P_{\mathcal{S}}
$$

where $\bar{P}_{\mathcal{S}, m}, P_{\mathcal{S}}$ are the desired mechanical and the electrical output power of the generator, $H$ is the inertia constant and $G_{\mathrm{PM}}$ contains the dynamics of the prime mover. The resulting transfer function is:

$$
\begin{gathered}
\omega_{\mathcal{S}}=\left[\begin{array}{ll}
G_{\mathcal{S}, m} & G_{\mathcal{S}, e}
\end{array}\right]\left[\begin{array}{c}
\bar{P}_{\mathcal{S}, m} \\
-P_{\mathcal{S}}
\end{array}\right] \\
G_{\mathcal{S}, m}=G_{\mathrm{PM}} \frac{\bar{\omega}}{2 H s}, \quad G_{\mathcal{S}, e}=\frac{\bar{\omega}}{2 H s}
\end{gathered}
$$

The voltage at an SG bus is commonly tightly regulated by the internal AVR (Automatic Voltage Regulator) of the machine. The closed-loop response of the AVR can again be formulated as a transfer function:

$$
U_{\mathcal{S}}=G_{\mathcal{S}, U} \bar{U}_{\mathcal{S}}
$$


where $\bar{U}_{\mathcal{S}}$ is the desired SG bus voltage magnitude. A simple way to model the prime mover and AVR dynamics is through the following first-order dynamics:

$$
G_{\mathrm{PM}}=\frac{1}{\tau_{m} s+1}, \quad G_{\mathcal{S}, U}=\frac{1}{\tau_{U} s+1}
$$

where $\left[\tau_{m}, \tau_{U}\right]$ are the time constants of the prime mover and AVR.

\section{E. Complete Model}

Combining the transfer function models established in the previous sections, it is now possible to construct the complete dynamic phasor model of a grid with any number of VSIs, SGs and constant power loads. The closed-loop block diagram of the complete model is shown in Fig. 1. In a classical formulation the controller transfer function matrices $K_{\mathcal{I}}, K_{\mathcal{S}, U}, K_{\mathcal{S}, m}$ would be the droop controllers. $G_{\text {sens }}$ is a $(2 p+2 q) \times(2 p+2 q)$ diagonal transfer function matrix containing the sensor dynamics.

In order to achieve a form suitable for control design, the plant is rewritten as single transfer function matrix $G_{\text {comp }}$ (as indicated in Fig. 1), with the in- and outputs corresponding to the classical droop control scheme. The inputs of $G_{\text {comp }}$ are the setpoints of the VSI frequency, the VSI and SG voltage magnitude and the SG mechanical input power. The outputs are the VSI active power, the VSI and SG reactive power and the SG frequency. To achieve this, first $G_{\text {grid }}$ from (14) is partitioned and reordered such that the following transfer functions are obtained:

$$
\begin{aligned}
{\left[\begin{array}{c}
P_{\mathcal{I}} \\
Q_{\mathcal{I}} \\
P_{\mathcal{S}} \\
Q_{\mathcal{S}}
\end{array}\right] } & =\left[\begin{array}{ll}
G_{\text {grid }}^{u \rightarrow P_{\mathcal{I}}} & G_{\text {grid }}^{\omega_{\mathcal{S}} \rightarrow P_{\mathcal{I}}} \\
G_{\text {grid }}^{u \rightarrow Q_{\mathcal{I}}} & G_{\text {grid }}^{\omega_{\mathcal{S}} \rightarrow Q_{\mathcal{I}}} \\
G_{\text {grid }}^{u \rightarrow P_{\mathcal{S}}} & G_{\text {grid }}^{\omega_{\mathcal{S}} \rightarrow P_{\mathcal{S}}} \\
G_{\text {grid }}^{u \rightarrow Q_{\mathcal{S}}} & G_{\text {grid }}^{\omega_{\mathcal{S}} \rightarrow Q_{\mathcal{S}}}
\end{array}\right]\left[\begin{array}{c}
u \\
\omega_{\mathcal{S}}
\end{array}\right] \\
u & =\left[\begin{array}{lll}
\omega_{\mathcal{I}} & U_{\mathcal{I}} & U_{\mathcal{S}}
\end{array}\right]^{T}
\end{aligned}
$$

Now, the single block transfer function of the plant can be obtained as follows:

$$
\begin{gathered}
{\left[\begin{array}{c}
P_{\mathcal{I}} \\
Q_{\mathcal{I}} \\
Q_{\mathcal{S}} \\
\omega_{\mathcal{S}}
\end{array}\right]=G_{\text {comp }}\left[\begin{array}{c}
\bar{u} \\
\bar{P}_{\mathcal{S}, m}
\end{array}\right], \bar{u}=\left[\begin{array}{ccc}
\bar{\omega}_{\mathcal{I}} & \bar{U}_{\mathcal{I}} & \bar{U}_{\mathcal{S}}
\end{array}\right]^{T}} \\
G_{\text {comp }}=G_{\text {sens }}\left[\begin{array}{ll}
G_{11} & G_{12} \\
G_{21} & G_{22} \\
G_{31} & G_{32} \\
G_{41} & G_{42}
\end{array}\right]\left[\begin{array}{ccc}
G_{\mathcal{I}} & 0 & 0 \\
0 & G_{\mathcal{S}, U} & 0 \\
0 & 0 & I
\end{array}\right]
\end{gathered}
$$

where $I$ is the identity matrix, and:

$$
\begin{aligned}
G_{11} & =\left(G_{\text {grid }}^{u \rightarrow P_{\mathcal{I}}}-G_{\text {grid }}^{\omega_{\mathcal{S}} \rightarrow P_{\mathcal{I}}} G_{\mathcal{S}, e} G_{51}\right) \\
G_{12} & =G_{\text {grid }}^{\omega_{\mathcal{S}} \rightarrow P_{\mathcal{I}}}\left(G_{\mathcal{S}, m}-G_{\mathcal{S}, e} G_{52}\right) \\
G_{21} & =G_{\text {grid }}^{u \rightarrow Q_{\mathcal{I}}}-G_{\text {grid }}^{\omega_{\mathcal{S}} \rightarrow Q_{\mathcal{I}}} G_{\mathcal{S}, e} G_{51} \\
G_{22} & =G_{\text {grid }}^{\omega_{\mathcal{S}} \rightarrow Q_{\mathcal{I}}}\left(G_{\mathcal{S}, m}-G_{\mathcal{S}, e} G_{52}\right) \\
G_{31} & =G_{\text {grid }}^{u \rightarrow Q_{\mathcal{S}}}-G_{\text {grid }}^{\omega_{\mathcal{S}} \rightarrow Q_{\mathcal{S}}} G_{\mathcal{S}, e} G_{51} \\
G_{32} & =G_{\text {grid }}^{\omega_{\mathcal{S}} \rightarrow Q_{\mathcal{S}}}\left(G_{\mathcal{S}, m}-G_{\mathcal{S}, e} G_{52}\right) \\
G_{41} & =G_{\mathcal{S}, e} G_{51} \\
G_{42} & =G_{\mathcal{S}, m}-G_{\mathcal{S}, e} G_{52} \\
G_{51} & =\left(I+G_{\text {grid }}^{\omega_{\mathcal{S}} \rightarrow P_{\mathcal{S}}} G_{\mathcal{S}, e}\right)^{-1} G_{\text {grid }}^{u \rightarrow P_{\mathcal{S}}} \\
G_{52} & =\left(I+G_{\text {grid }}^{\omega_{\mathcal{S}} \rightarrow P_{\mathcal{S}}} G_{\mathcal{S}, e}\right)^{-1} G_{\text {grid }}^{\omega_{\mathcal{S}} \rightarrow P_{\mathcal{S}}} G_{\mathcal{S}, m}
\end{aligned}
$$

Written in this form, the matrix transfer function $G_{\text {comp }}$ can readily be used for small-signal stability analysis and control design. It describes accurately the electromagnetic and electromechanic dynamics of the grid, and common performance criteria (e.g. load sharing, maximum frequency excursion) can easily be formulated using standard robust control design techniques.

\section{Control Design Method}

In this section a novel approach to fixed-structure, robust control design based on the frequency response of multivariable systems and convex optimization is presented. For reasons of space, only an abbreviated version of the method will be presented in this section. A full theoretical exposition can be found in [31].

\section{A. Frequency Response Data}

The system to be controlled is a Linear Time-Invariant multivariable (LTI-MIMO) strictly proper system represented by its frequency response $G(j \omega) \in \mathbb{C}^{n \times m}$, where $m$ is the number of inputs and $n$ is the number of outputs. $G(j \omega)$ is assumed to be bounded in all frequencies except for a set $B_{g}$ including a finite number of frequencies that correspond to the poles of $G$ on the imaginary axis. Further, define $\omega \in \Omega$ with:

$$
\Omega=\left\{\omega \mid-\frac{\pi}{T_{s}} \leq \omega \leq \frac{\pi}{T_{s}}\right\} \backslash B_{g}
$$

where $T_{s}$ is the sampling time of the controller.

\section{B. Controller Structure}

As the design is based upon frequency-domain methods, it is possible to directly design discrete-time controllers using the frequency response of either discrete- or continuous-time plants. A fixed-structure discrete-time matrix transfer function controller is considered. The controller is defined as $K(z)=$ $X(z) Y(z)^{-1}$, where $X(z)$ and $Y(z)$ are polynomial matrices in $z$. We have:

$$
\begin{aligned}
& X(z)=X_{p} z^{p}+\cdots+X_{1} z+X_{0} \\
& Y(z)=I z^{p}+\cdots+Y_{1} z+Y_{0}
\end{aligned}
$$




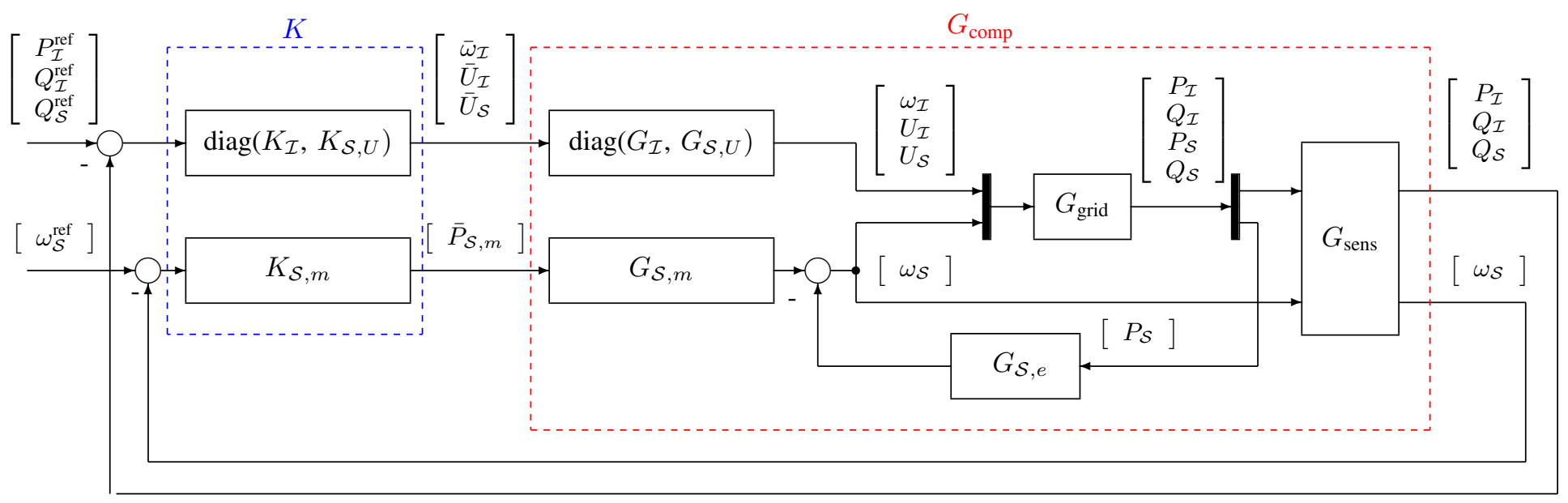

Fig. 1. Block diagram of the full dynamic phasor model.

where $X_{i} \in \mathbb{R}^{m \times n}$ for $i=0, \ldots, p$ and $Y_{i} \in \mathbb{R}^{n \times n}$ for $i=0, \ldots, p-1$ contain the controller parameters. Note that $Y\left(e^{j \omega}\right)$ must be invertible $\forall \omega \in \Omega$.

\section{Control performance}

The control performance is defined as constraints on the norm of weighted sensitivity functions. For example, a typical performance specification for good tracking performance and output disturbance rejection is to minimize the following norm:

$$
\min _{X, Y}\left\|W_{1} S\right\|_{\infty}
$$

where $S=(I+G K)^{-1}$ is the sensitivity function and $W_{1}$ is the performance weight. In order to limit the control input and prevent chattering, the following constraint can be considered:

$$
\left\|W_{2} K S\right\|_{\infty}<1
$$

where $K S$ is the input sensitivity and $W_{2}$ is the input weight. For a stable system $H(z)$, the infinity-norm is defined as:

$$
\|H\|_{\infty}=\sup _{\omega} \bar{\sigma}\left[H\left(e^{j \omega}\right)\right]
$$

where $\bar{\sigma}$ denotes the maximum singular value. Note that the weighting filters can be continuous- or discrete-time transfer functions, or just simple numerical weights. Also, note that reversely the boundedness of the spectral norm of $H$ does not guarantee the stability of $H$.

\section{Convex Approximation}

The performance specifications described in the previous section lead to a non-convex optimization problem. In order to convexify this problem, the performance constraints can be represented by a set of convex-concave constraints, and can then be approximated by an inner convex approximation based on the linearization of the concave parts.
As an illustrative example, the optimization problem in (28) can be written as:

$$
\min _{X, Y} \gamma
$$

subject to:

$$
\left(W_{1} S\right)^{*}\left(W_{1} S\right)<\gamma I, \quad \forall \omega \in \Omega
$$

where $(\cdot)^{*}$ denotes the complex conjugate transpose. Replacing $K$ with $X Y^{-1}$ in the constraint gives:

$$
\left[W_{1} Y(Y+G X)^{-1}\right]^{*}\left[W_{1} Y(Y+G X)^{-1}\right]<\gamma I, \forall \omega \in \Omega
$$

Note that the dependency in $\omega$ has been omitted for $G(j \omega), K\left(e^{j \omega}\right)$ and $W_{1}\left(e^{j \omega}\right)$ in order to simplify the notation. Multiplying both sides from the right by $(Y+G X)$, and from the left by its complex conjugate, leads to the following matrix inequality:

$$
\left[W_{1} Y\right]^{*} \gamma^{-1}\left[W_{1} Y\right]-(Y+G X)^{*}(Y+G X)<0, \forall \omega \in \Omega
$$

which is a constraint on the difference between two quadratic terms (a convex-concave constraint). In order to convexify the constraint, the second quadratic term is linearized using the following property:

$$
P^{*} P \geq P^{*} P_{c}+P_{c}^{*} P-P_{c}^{*} P_{c}
$$

where $P=Y+G X$ and $P_{c} \in \mathbb{C}^{n \times m}$ is any known complex matrix. Let $P_{c}=Y_{c}+G X_{c}$, where $K_{c}=X_{c} Y_{c}^{-1}$ is an initial controller. Using the Schur complement, the constraint in (33) can then represented by a linear matrix inequality:

$$
\left[\begin{array}{cc}
P^{*} P_{c}+P_{c}^{*} P-P_{c}^{*} P_{c} & \left(W_{1} Y\right)^{*} \\
W_{1} Y & \gamma I
\end{array}\right]>0, \forall \omega \in \Omega
$$

This convex constraint is a sufficient condition for the spectral constraint in (31) for any choice of $K_{c}=X_{c} Y_{c}^{-1}$. However, this constraint will not necessarily represent a convex set of stabilizing controllers. In fact, every unstable system with no pole on the stability boundary has a bounded spectral norm. The conditions on the linearization of the constraints such that the closed-loop stability can be guaranteed is given in the following theorem. 
Theorem 1: Given a plant model $G$, an initial stabilizing controller $K_{c}=X_{c} Y_{c}^{-1}$ with $\operatorname{det}\left(Y_{c}\right) \neq 0, \forall \omega \in \Omega$, and feasible solutions $X$ and $Y$ to the following LMI,

$$
(Y+G X)^{*}\left(Y_{c}+G X_{c}\right)+\left(Y_{c}+G X_{c}\right)^{*}(Y+G X)>0
$$

for all $\omega \in \Omega$, then the controller $K=X Y^{-1}$ stabilizes the closed-loop system if

1) $\operatorname{det}(Y) \neq 0, \forall \omega \in \Omega$.

2) The initial controller $K_{c}$ and the final controller $K$ share the same poles on the stability boundary, i.e. $\operatorname{det}(Y)=$ $\operatorname{det}\left(Y_{c}\right)=0, \forall \omega \in B_{y}$.

Proof: The proof is given in [31].

Remark: A necessary and sufficient condition for $\operatorname{det}(Y) \neq$ 0 is $Y^{*} Y>0$. Since this constraint is concave, it can be linearized to obtain the following sufficient convex constraint:

$$
Y^{*} Y_{c}+Y_{c}^{*} Y-Y_{c}^{*} Y_{c}>0
$$

Furthermore, the condition in (36) is automatically fulfilled when considering an $H_{\infty}$ constraint on any closed-loop sensitivity function.

\section{E. Multimodel Uncertainty}

An important specification in power grids is that the controller should be robust towards structural changes in the grid, such as topology changes or generator outages. One way to incorporate this into the design process is as a multimodel uncertainty. A system that has different frequency responses in $g$ different operating points can be represented by a multimodel uncertainty set:

$$
\mathcal{G}\left(e^{j \omega}\right)=\left\{G_{1}\left(e^{j \omega}\right), G_{2}\left(e^{j \omega}\right), \ldots, G_{g}\left(e^{j \omega}\right)\right\}
$$

This can easily be included in the presented framework by formulating a different set of constraints for each model. Let $P_{i}=Y+G_{i} X$ and $P_{c_{i}}=Y_{c}+G_{i} X_{c}$. Again taking the sensitivity problem in (28) as an example, the convex formulation of this problem including the stability constraint would be:

$$
\min _{X, Y} \gamma
$$

subject to:

$$
\begin{aligned}
& {\left[\begin{array}{cc}
P_{i}^{*} P_{c_{i}}+P_{c_{i}}^{*} P_{i}-P_{c_{i}}^{*} P_{c_{i}} & \left(W_{1} Y\right)^{*} \\
W_{1} Y & \gamma I
\end{array}\right]>0} \\
& Y^{*} Y_{c}+Y_{c}^{*} Y-Y_{c}^{*} Y_{c}>0 \\
& \text { for } i=1, \ldots, g \quad ; \quad \forall \omega \in \Omega
\end{aligned}
$$

\section{F. Frequency Gridding and Iterative Algorithm}

The convex optimization problem formulated in the previous section contains an infinite number of constraints (i.e. $\forall \omega \in \Omega$ ) and is called a semi-infinite problem. A common approach to handle this type of constraints is to choose a reasonably large set of frequency samples $\Omega_{N}=\left\{\omega_{1}, \ldots, \omega_{N}\right\}$ with:

$$
\omega_{1} \geq 0, \quad \omega_{N}=\frac{\pi}{T_{s}}
$$

and replace the constraints with a finite set of constraints at each of the given frequencies. The complexity of the problem scales linearly with the number of constraints, therefore $N$ can be chosen relatively large. Since all constraints are applied to Hermitian matrices, the constraints for the negative frequencies between $-\pi / T_{s}$ and zero will be automatically satisfied.

Any LMI solver can be used to solve the optimization problem and calculate a suboptimal controller $K$ around a stabilizing initial controller $K_{c}$. Since an inner convex approximation of the original non-convex problem is solved, the performance criterion for $K$ may be quite far from the optimal value. The solution is to use an iterative approach that solves the optimization problem multiple times, using the calculated controller $K$ of the previous step as the new initial controller $K_{c}$. This choice always guarantees closed-loop stability (assuming the initial choice of $K_{c}$ is stabilizing). Since the objective function is non-negative and non-increasing, the iteration converges to a local optimal solution of the original non-convex problem. The iterative process can be stopped once the change in the performance criterion is sufficiently small.

\section{Simulation Example}

In order to demonstrate their potential and versatility, the dynamic phasor model and the control design method are applied in a comprehensive simulation example. Using a model based on a real-world medium-voltage distribution grid, three distinct scenarios with decentralized and distributed control architectures are investigated. The results are evaluated in continuous-time nonlinear simulation using Simulink and the Simpower toolbox.

\section{A. Example Grid}

A grid model based on the three-phase islanded Subnetwork 1 of the CIGRE benchmark medium voltage distribution network is used [32]. The network is a meshed network with mixed lines consisting of 11 buses (see Fig. 2).

The following modifications are made compared to the original system: the nominal phase-to-phase voltage is set to $600 \mathrm{~V}$. Only 2 VSI-interfaced battery storage units connected to buses 5, 9 and a SG connected to bus 10 are considered. The photovoltaics are assumed to operate in maximum power point tracking mode and are absorbed into the loads. The loads at buses 1, 5, 9 and 10 are neglected. Since the grid is running in islanded mode, the loads and power ratings of the generation units are scaled such that nominal generation and load is at an equilibrium. To prevent the dynamics from being dominated by a single generation unit, the power ratings are in a similar range for all three units. The line impedances of the grid are taken from [32], the other relevant parameters are listed in Table I, including the values of the initial droop controller. $R_{f}, L_{f}$ are the resistance and inductance of the VSI $L$-type output filters, $R_{o}, L_{o}$ the resistance and inductance of the internal impedance of the SG. Finally, the line between buses 9 and 10 can be opened or closed, leading to a change in topology.

\section{B. Part 1: Improved Primary Control Performance}

In a first step, the goal is to design a decentralized controller to improve the frequency and voltage transient performance, 


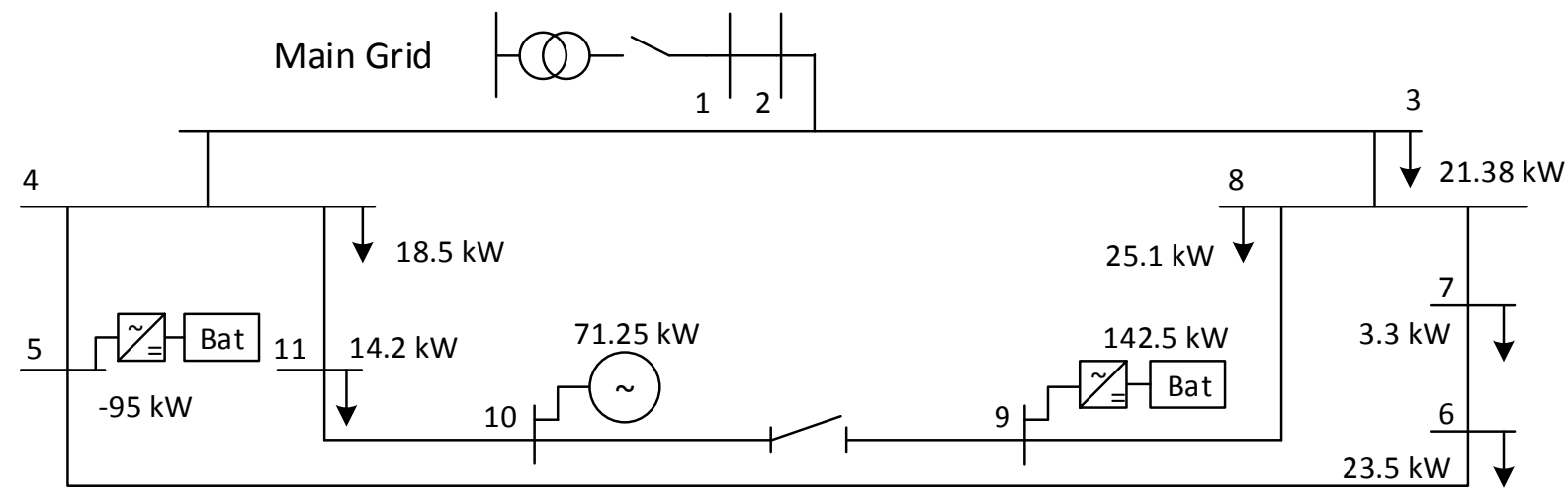

Fig. 2. Model adapted from [32] with 11 buses, 3 inverter-interfaced batteries and 6 loads. The sign $\downarrow$ denotes the loads.

TABLE I

TEST GRID PARAMETERS

\begin{tabular}{|c|c|}
\hline Base Values & $S_{\text {base }}=475 \mathrm{kVA}, U_{\text {base }}=600 \mathrm{~V}, \mathrm{f}=50 \mathrm{~Hz}$ \\
\hline \multicolumn{2}{|l|}{ Batteries } \\
\hline bus & [5 9] \\
\hline$R_{f}=0.01 \Omega$ & $L_{f}=10^{-4} \mathrm{H}$ \\
\hline$\tau_{\omega}=5 \cdot 10^{-4}$ & $\tau_{U}=5 \cdot 10^{-4}$ \\
\hline $\bar{P}_{\mathcal{I}}$ & {$\left[\begin{array}{ll}-0.2 & 0.3\end{array}\right] \mathrm{pu}$} \\
\hline $\bar{Q}_{\mathcal{I}}$ & {$[0.1263-0.0905] \mathrm{pu}$} \\
\hline$k_{p}^{\mathrm{VSI}}$ & $\operatorname{diag}(2.625,1.75) \frac{\mathrm{Hz}}{\mathrm{pu}}$ \\
\hline$k_{q}^{\mathrm{VSI}}$ & $\operatorname{diag}(20,20) \frac{\mathrm{v}}{\mathrm{nu}}$ \\
\hline \multicolumn{2}{|l|}{ Diesel Generator } \\
\hline$R_{o}=1.8 \cdot 10^{-3} \Omega$ & $L_{o}=8.2 \cdot 10^{-7} \mathrm{H}$ \\
\hline $\mathrm{H}$ & 1.5 \\
\hline$\tau_{m}=0.1$ & $\tau_{U}=0.1$ \\
\hline $\bar{P}_{\mathcal{S}}$ & {$[0.15] \mathrm{pu}$} \\
\hline $\bar{Q}_{\mathcal{S}}$ & {$[-0.0105] \mathrm{pu}$} \\
\hline$k_{p}^{\mathrm{SG}}$ & $\frac{1}{3.5} \frac{\mathrm{pu}}{\mathrm{Hz}}$ \\
\hline$k_{q}^{\mathrm{SG}}$ & $20 \frac{\mathrm{V}}{\mathrm{pu}}$ \\
\hline \multicolumn{2}{|l|}{ Loads } \\
\hline bus & {$\left[\begin{array}{llllll}3 & 4 & 6 & 7 & 8 & 11\end{array}\right]$} \\
\hline$P_{\mathcal{L}}$ & {$\left[\begin{array}{llllll}0.045 & 0.039 & 0.05 & 0.007 & 0.053 & 0.03\end{array}\right] \mathrm{pu}$} \\
\hline$Q_{\mathcal{L}}$ & {$\left[\begin{array}{lllllll}0 & 0 & 0 & 0 & 0 & 0\end{array}\right] \mathrm{pu}$} \\
\hline
\end{tabular}

while maintaining proportional active power sharing. Using the dynamic phasor model, the plant is formed as described in Section II-E. The VSI output filters and the internal impedance of the SG are lumped with the line impedances. The line 9-10 is assumed to be open. For the sensor dynamics, the active and reactive power measurements are filtered using a firstorder low-pass filter with a cutoff frequency of $5 \mathrm{~Hz}$. The SG frequency measurement is assumed to not have any sensor dynamics.

1) Performance Specifications: The closed-loop response of the initial droop controller exhibits long settling times and exorbitant ringing. In order to address these issues, a 4thorder decentralized controller with a sampling time of $T_{s}=$ $1 \mathrm{~ms}$ is designed that guarantees stability and proportional power sharing, while significantly improving the transient performance. The plant is chosen as $G=G_{\text {comp }}$, which has been defined in Section II-E. Furthermore, all inputs and outputs of the plant are normalized to per unit, using the base power and frequency given in Table I.

As objective function, the infinity norm of the weighted sensitivity $\left\|W_{1} S\right\|_{\infty}$ is minimized, where the weighting filter is chosen as $W_{1}=I$. Also, the maximum input sensitivity is constrained, and a roll-off at high frequencies is added to prevent undesired fast oscillations in the inputs:

$$
\left\|W_{2} K S\right\|<1
$$

with

$$
W_{2}^{-1}=10 \operatorname{diag}\left(k_{p}^{\mathrm{VSI}}, k_{q}^{\mathrm{VSI}}, k_{q}^{\mathrm{SG}}, k_{p}^{\mathrm{SG}}\right) B
$$

The desired droop gains are used to normalize the input sensitivity accordingly. $B$ is a second-order discrete-time Butterworth low-pass filter with a cutoff frequency of $500 \mathrm{rad} / \mathrm{s}$.

Proportional power sharing is maintained by constraining the steady-state gains of the new controller to be equal to the droop gains. The constraint can be formulated as follows:

$$
X(1) Y(1)^{-1}=\operatorname{diag}\left(k_{p}^{\mathrm{VSI}}, k_{q}^{\mathrm{VSI}}, k_{q}^{\mathrm{SG}}, k_{p}^{\mathrm{SG}}\right)
$$

which can be expressed as:

$$
\sum_{i=1}^{4} X_{i}=\operatorname{diag}\left(k_{p}^{\mathrm{VSI}}, k_{q}^{\mathrm{VSI}}, k_{q}^{\mathrm{SG}}, k_{p}^{\mathrm{SG}}\right) \sum_{i=1}^{4} Y_{i}
$$

As stabilizing initial controller, the original droop controller is used:

$$
X_{c}(z)=\operatorname{diag}\left(k_{p}^{\mathrm{VSI}}, k_{q}^{\mathrm{VSI}}, k_{q}^{\mathrm{SG}}, k_{p}^{\mathrm{SG}}\right) \quad ; \quad Y_{c}(z)=I
$$

The problem is sampled using 600 logarithmically-spaced frequency points in the interval $\Omega_{N}=\left\{1,10^{3} \pi\right\} \mathrm{rad} / \mathrm{s}$ where the upper limit is the Nyquist frequency of the controller. 
Finally, using the method presented in Section III, the control design problem is reformulated as a convex optimization problem:

$$
\min _{X, Y} \gamma
$$

subject to:

$$
\begin{aligned}
& {\left[\begin{array}{cc}
P^{*} P_{c}+P_{c}^{*} P-P_{c}^{*} P_{c} & \left(W_{1} Y\right)^{*} \\
W_{1} Y & \gamma I
\end{array}\right]\left(j \omega_{n}\right)>0} \\
& {\left[\begin{array}{cc}
P^{*} P_{c}+P_{c}^{*} P-P_{c}^{*} P_{c} & \left(W_{2} X\right)^{*} \\
W_{2} X & I
\end{array}\right]\left(j \omega_{n}\right)>0} \\
& {\left[Y_{c}^{*} Y+Y^{*} Y_{c}-Y_{c}^{*} Y_{c}\right]\left(j \omega_{n}\right)>0} \\
& \sum_{i=1}^{4} X_{i}=\operatorname{diag}\left(k_{p}, k_{q}\right) \cdot \sum_{i=1}^{4} Y_{i} \\
& \omega_{n} \in \Omega_{N}
\end{aligned}
$$

where the first constraint is for the objective function, the second constraint is to limit $K S$, the third constraint is the stability condition from Eq. 37, and the fourth constraint is for proportional power sharing. The optimization problem is formulated in Matlab using Yalmip [33], and solved with Mosek [34]. The algorithm converges within 8 iterations, which takes less than 5 minutes on a standard desktop computer in our simple implementation. The achieved maximum singular values of the sensitivity and input sensitivity of the original droop controller and the new controller are shown in Fig. 3. It can be seen that the resonance peaks in the sensitivity have been successfully attenuated, and that the input sensitivity rolls off at high frequencies.

2) Simulation Results: To validate the performance, the grid is simulated in Simulink using the Simpower toolbox, and the performance of the new controller is compared with the original droop controller. In Fig. 4 the evolution of the frequency of the generators is shown after the active power load at bus 3 is stepped up by $47.5 \mathrm{~kW}$ at $\mathrm{t}=1 \mathrm{~s}$. The improved primary controller reduces the settling time from $0.9 \mathrm{~s}$ to $0.2 \mathrm{~s}$, an improvement of $80 \%$ from the droop controller. The plots also show that the ringing is successfully damped.

The active and reactive output power of the generators is shown in Figs. 5 and 6. The settling time of the new controller is again significantly improved, and the transients are smoother with minimal ringing.

\section{Part 2: Robustness Towards Topology Change}

For the second part of this example, the line between buses 9 and 10 is closed, leading to a change in topology. While the controller designed in Part 1 satisfies the desired performance specifications as long as the line is open, there is no guarantee of stability or performance when it is closed. Indeed, the maximum singular value plots of the dynamic phasor models of the two configurations (see Fig. 7) are noticeably different, meaning that the grid dynamics effectively change when the line is closed.

While the stability of the previous controller with line 9-10 closed could be determined a posteriori, a preferable approach is to directly consider both models during the design process.

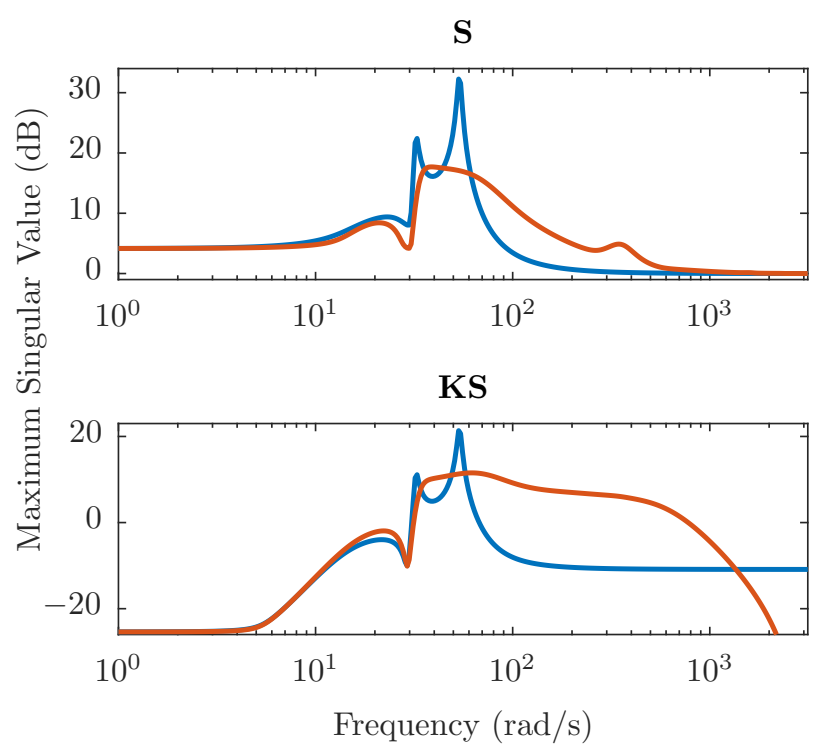

Fig. 3. Maximum singular value plots of the sensitivity $S$ and input sensitivity $K S$. Blue is the droop controller, red is the new controller.
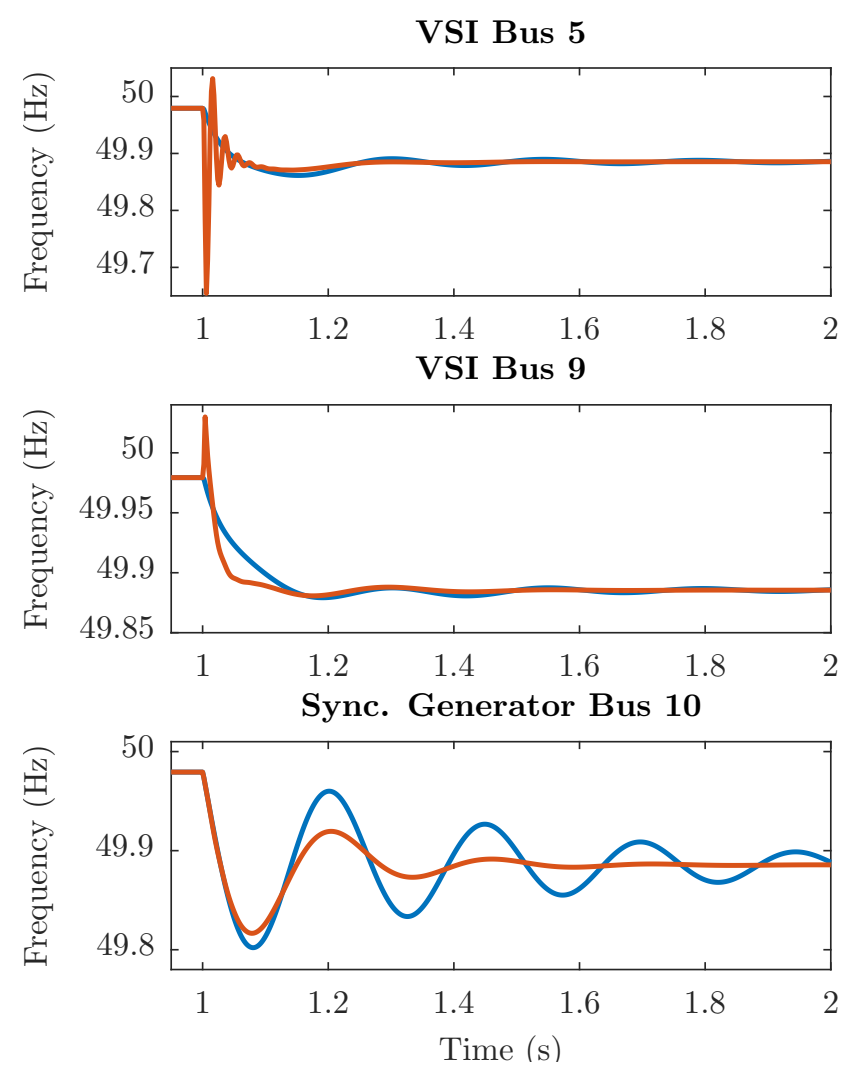

Fig. 4. Generator frequency after a load step. Blue is the droop controller, red is the new primary controller. 

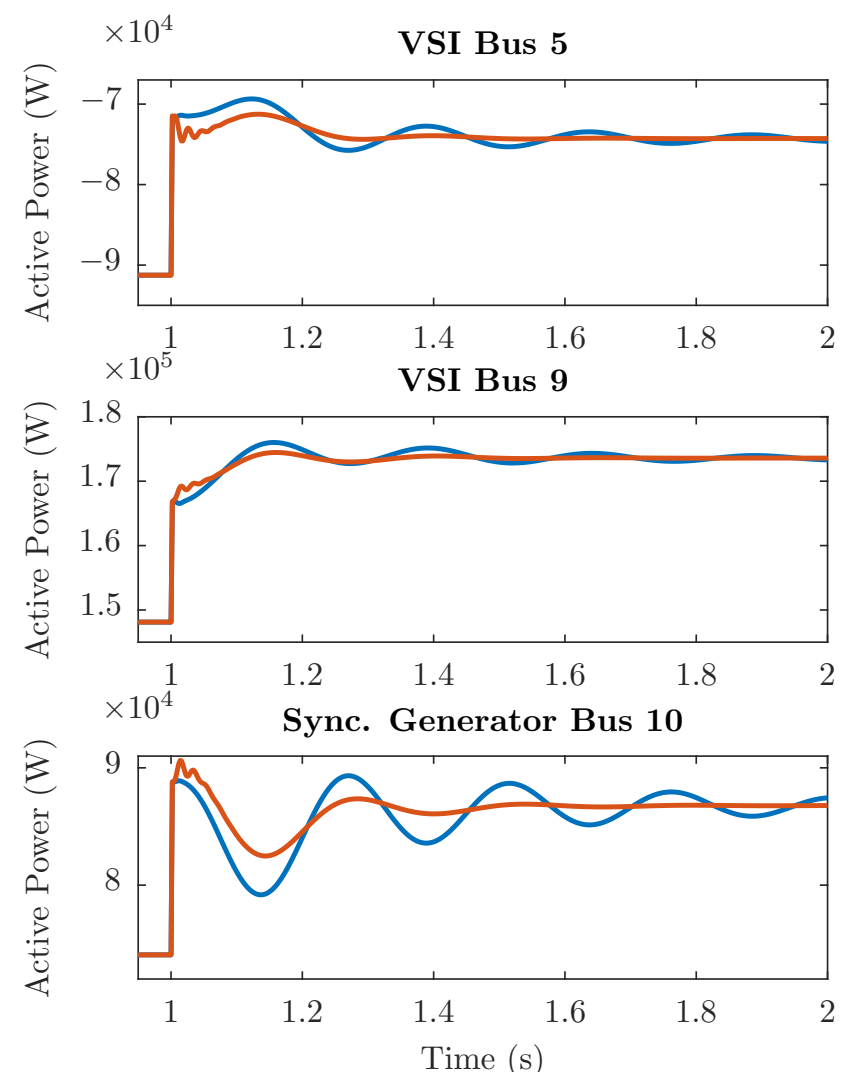

Fig. 5. Generator active output power after a load step. Blue is the droop controller, red is the new primary controller.

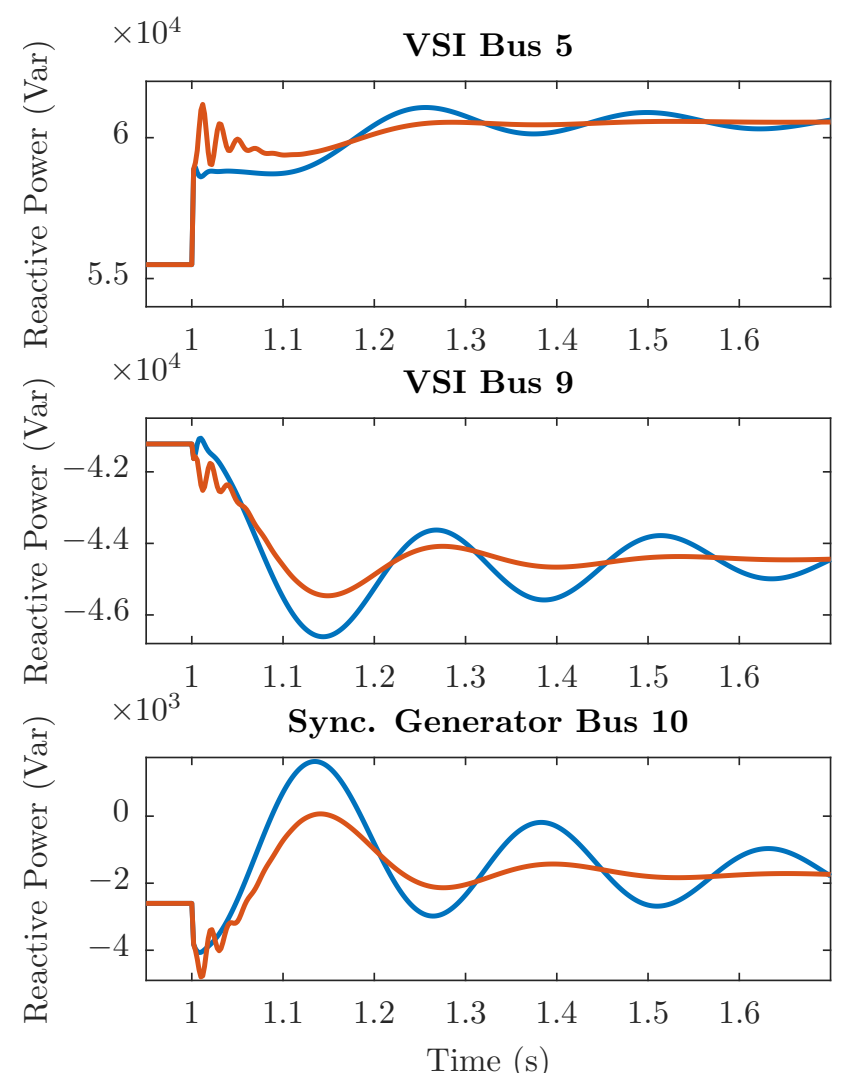

Fig. 6. Generator reactive output power after a load step. Blue is the droop controller, red is the new primary controller.

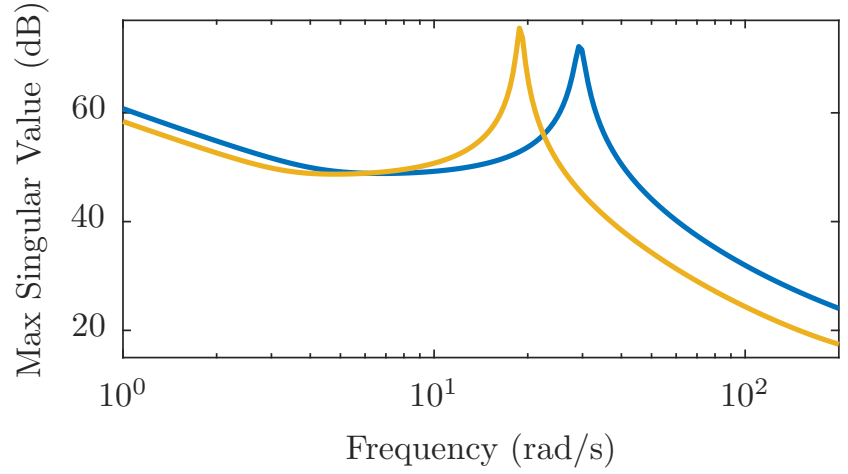

Fig. 7. Maximum singular value plots of the plant models $G_{\text {comp }}$. Blue is with line 9-10 closed, yellow is with line 9-10 open.

This can be done by introducing a multimodel uncertainty to the control design problem, as described in Section III-E.

First, $G_{\text {comp }}$ is formed for line 9-10 open and closed respectively. The optimization problem in Eq. (45) is then solved for the multimodel case, using the same performance specifications and initial controller. The performance of the resulting controller after closing line 9-10 is evaluated in simulation. The frequency of the generation units after line 9-10 is closed at $\mathrm{t}=1 \mathrm{~s}$ is shown in Fig. 8 both for the multimodel controller and controller calculated in Part 1. It can be seen that the frequency transients of the VSIs are improved by the multimodel design. Also, stability and performance are guaranteed for both topologies by design.

\section{Part 3: Distributed Secondary Control}

While the controllers designed in Parts 1 and 2 improve the transient performance, as can be seen in Fig. 4 the lack of integral action still introduces a significant steady-state error in the frequency after a load change. The simplest solution for this issue would be to add decentralized integrators to the controller of each generation unit. However, in practice this approach is not feasible, as any bias in the measurements or control inputs would render the system unstable. Therefore, the controller structure is augmented with a distributed part that fulfills the task of traditional centralized secondary frequency control, but at a significantly higher bandwidth. In [11] a distributed integral control scheme is proposed that adjusts the active power setpoints of the generation units in order to eliminate the frequency error. However, no method to tune the controller gains is given. The presented control design method will be used to calculate the gains such that the integral action achieves a desired bandwidth, while guaranteeing closed-loop stability and performance. It is important to point out that the ability of our method to directly design discrete-time controllers is critical in this example. Whereas the continuoustime formulation of the distributed integral controller is stable for any choice of positive controller gains, in discrete-time decreasing the gains leads to a degradation of transient performance, and eventually instability. Therefore, being able to design the controller directly in discrete-time is a significant advantage. 

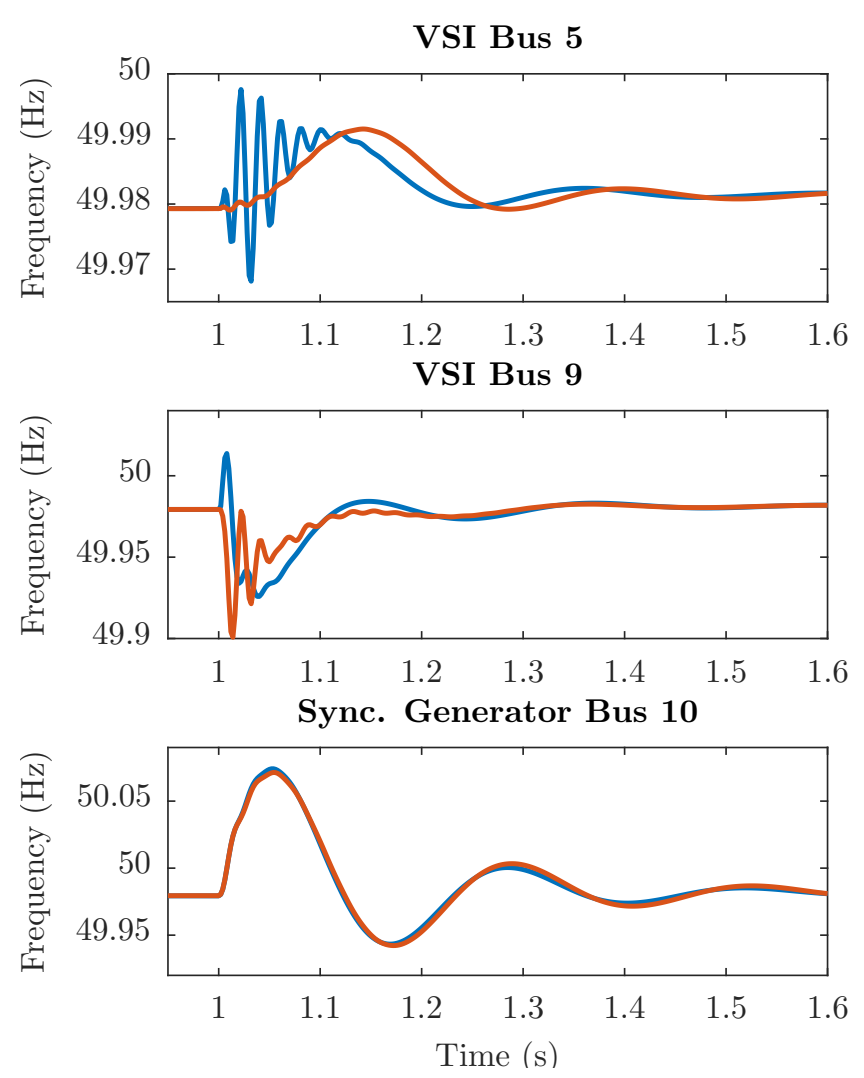

Fig. 8. Generator frequency after line $9-10$ is closed. Blue is the droop controller, red is the new primary controller.

According to [11], the distributed integral controller has the following dynamics:

$$
\begin{aligned}
& K_{I}\left[\begin{array}{c}
\delta \dot{P}_{\mathcal{I}} \\
\delta \dot{P}_{\mathcal{S}, m}
\end{array}\right]=K_{P}^{-1}\left[\begin{array}{c}
\omega_{\mathcal{I}} \\
\omega_{\mathcal{S}}
\end{array}\right]-L_{c} K_{P}\left[\begin{array}{c}
\delta P_{\mathcal{I}} \\
\delta P_{\mathcal{S}, m}
\end{array}\right] \\
& K_{P}=\operatorname{diag}\left(k_{p}^{\mathrm{VSI}}, k_{p}^{\mathrm{SG}}\right)
\end{aligned}
$$

where $K_{I}$ is a diagonal matrix containing the integral gains, $\delta P_{\mathcal{I}}, \delta P_{\mathcal{S}, m}$ are adjustments of the active power setpoints of the VSIs and SG, and $L_{c}$ is the Laplacian matrix of the communication graph. For this example, a non-full graph is assumed, where the generators at bus 5 and 10 are able to communicate with the generator at bus 9, but not directly with each other. Rewriting the controller as a discrete-time transfer function with a sampling time $T_{s}=1 \mathrm{~ms}$ results in:

$$
\begin{aligned}
{\left[\begin{array}{c}
\delta P_{\mathcal{I}} \\
\delta P_{\mathcal{S}, m}
\end{array}\right] } & =K_{\text {dist }}\left[\begin{array}{l}
\omega_{\mathcal{I}} \\
\omega_{\mathcal{S}}
\end{array}\right] \\
K_{\text {dist }} & =\left(K_{P} K_{I} z+\left(K_{P} L_{c} K_{P}-K_{P} K_{I}\right)\right)^{-1}
\end{aligned}
$$

The block diagram of the augmented system is shown in Fig. 9, where $K$ is the improved primary controller calculated in the Part 2 of this example.

1) Performance Specifications: In order to design the gains $K_{I}$ according to classical performance specifications, the plant $G_{\text {sec }}$ is formulated as follows:

$$
\left[\begin{array}{c}
\omega_{\mathcal{I}} \\
\omega_{\mathcal{S}}
\end{array}\right]=G_{\mathrm{sec}}\left[\begin{array}{c}
\delta P_{\mathcal{I}} \\
\delta P_{\mathcal{S}, m}
\end{array}\right]=\left[\begin{array}{cc}
G_{\mathrm{sec}}^{11} & G_{\mathrm{sec}}^{12} \\
G_{\mathrm{sec}}^{21} & G_{\mathrm{sec}}^{22}
\end{array}\right]\left[\begin{array}{c}
\delta P_{\mathcal{I}} \\
\delta P_{\mathcal{S}, m}
\end{array}\right]
$$

$G_{\mathrm{sec}}$

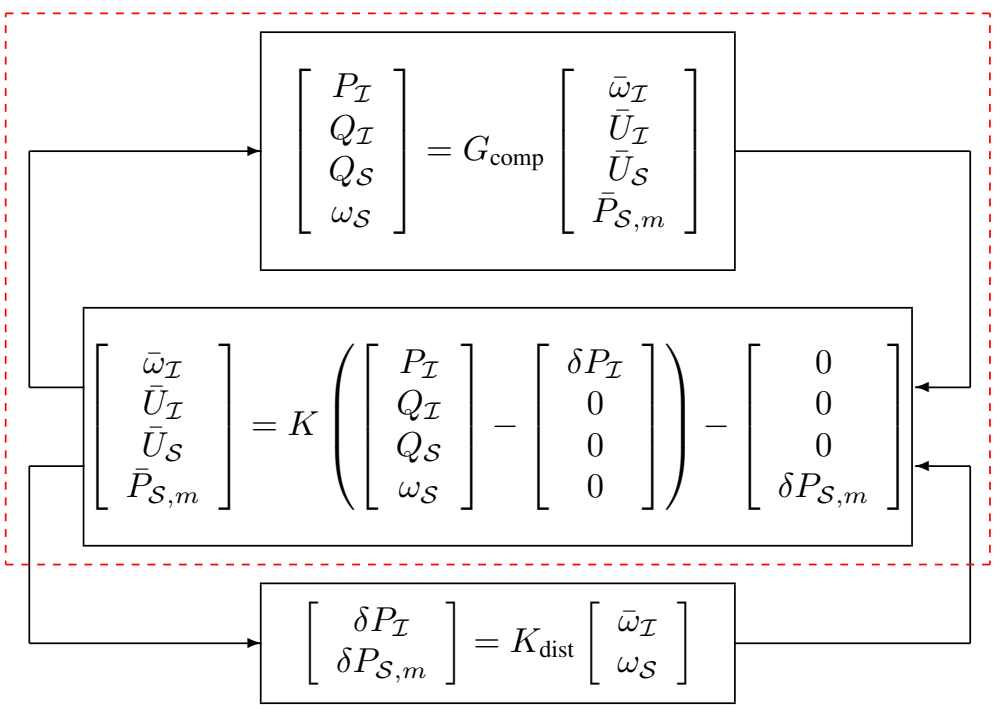

Fig. 9. Block diagram of the distributed secondary controller.

with

$$
\begin{array}{ll}
G_{\mathrm{sec}}^{11}=K\left(I+K G_{\mathrm{comp}}\right)^{-1} & G_{\mathrm{sec}}^{12}=\left(I+K G_{\mathrm{comp}}\right)^{-1} \\
G_{\mathrm{sec}}^{21}=\left(I+G_{\mathrm{comp}} K\right)^{-1} & G_{\mathrm{sec}}^{22}=G_{\mathrm{comp}}\left(I+G_{\mathrm{comp}} K\right)^{-1}
\end{array}
$$

A straightforward choice for the controller structure is:

$$
X=I, Y=K_{\text {dist }}^{-1}
$$

where $Y$ is linear in $K_{I}$. As a design objective, the gains in $K_{I}$ are minimized. As (47) depends on the inverse of $K_{I}$, this in turn maximizes the bandwidth of the integral action. Additionally, a constraint on the weighted sensitivity is introduced to maintain a smooth transient:

$$
\left\|W_{1} S\right\|<1, W_{1}=0.2 I
$$

with $S=\left(I+G_{\mathrm{sec}} K_{\mathrm{dist}}\right)^{-1}$. The initial controller is formed with $K_{I}=\operatorname{diag}\left(10^{3}, 10^{3}, 10^{3}\right)$, which leads to a stabilizing controller with a very low bandwidth. The problem is sampled using 600 logarithmically-spaced frequency points in the interval $\Omega_{N}=\left\{1,10^{3} \pi\right\} \mathrm{rad} / \mathrm{s}$ where the upper limit is the Nyquist frequency of the controller. The resulting convex optimization problem is as follows:

$$
\min _{K_{I}} \gamma
$$

subject to:

$$
\begin{aligned}
& K_{I}<\gamma I \\
& {\left[\begin{array}{cc}
P^{*} P_{c}+P_{c}^{*} P-P_{c}^{*} P_{c} & \left(W_{1} Y\right)^{*} \\
W_{1} Y & \gamma I
\end{array}\right]\left(j \omega_{n}\right)>0} \\
& {\left[Y_{c}^{*} Y+Y^{*} Y_{c}-Y_{c}^{*} Y_{c}\right]\left(j \omega_{n}\right)>0} \\
& \omega_{n} \in \Omega_{N}
\end{aligned}
$$

2) Simulation Results: As before, the resulting controller is evaluated in Simulink using the Simpower toolbox. In Fig. 10 a comparison of the evolution of frequency of the generators is shown after the active power load at bus 3 is stepped up by $47.5 \mathrm{~kW}$ at $\mathrm{t}=1 \mathrm{~s}$. With the addition of the distributed secondary controller, it can be seen that the 
VSI Bus 5
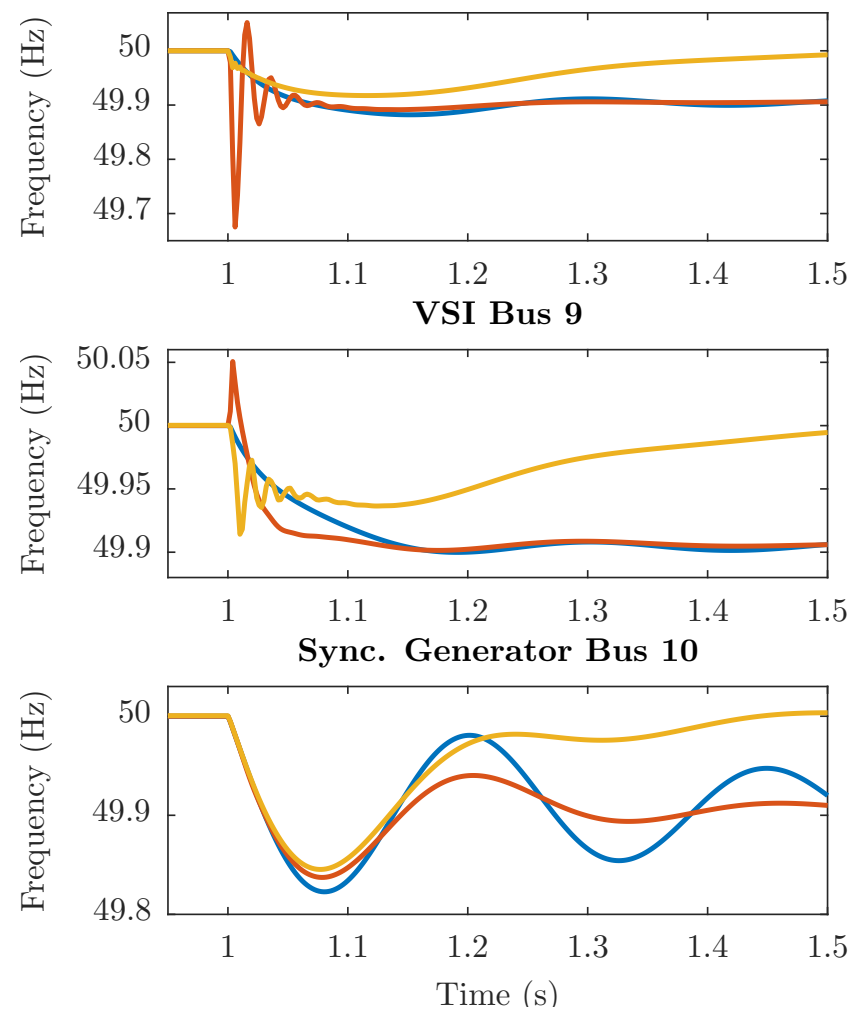

Fig. 10. Generator frequency after a load step. Blue is the droop controller, red is the improved primary controller, yellow is with the distributed controller.

frequency is returned to the nominal value within $0.5 \mathrm{~s}$ and with no overshoot. The transient response of the droop and improved primary controller are also plotted for comparison, showing that the settling time of the latter is similar to that of the distributed controller.

The active output power of the generators is shown in Fig. 11. With the distributed controller, the output power takes longer to settle at the new steady-state than with the improved primary controller. However, the settling time is still significantly smaller than that of the droop controller, and the transients are smooth with minimal ringing.

\section{CONCLUSION}

A novel dynamic phasor model for low- and mediumvoltage power grids has been developed. It is a pure frequencydomain model that accurately represents the electromagnetic and electromechanic dynamics of lines, inverters, synchronous machines and constant power loads. The model was then successfully used to design fixed-structure multivariable robust controllers based on a new frequency-domain control design method. Simulation results demonstrate the potential of the approach to guarantee frequency and voltage transient stability for a broad class of systems, while also achieving various desired performance specifications. The method is applicable to a wide spectrum of scenarios thanks to the general formulation of the performance objectives, allowing for a solution tailored to the specific problem at hand. In a next step, the model will be expanded to encompass more
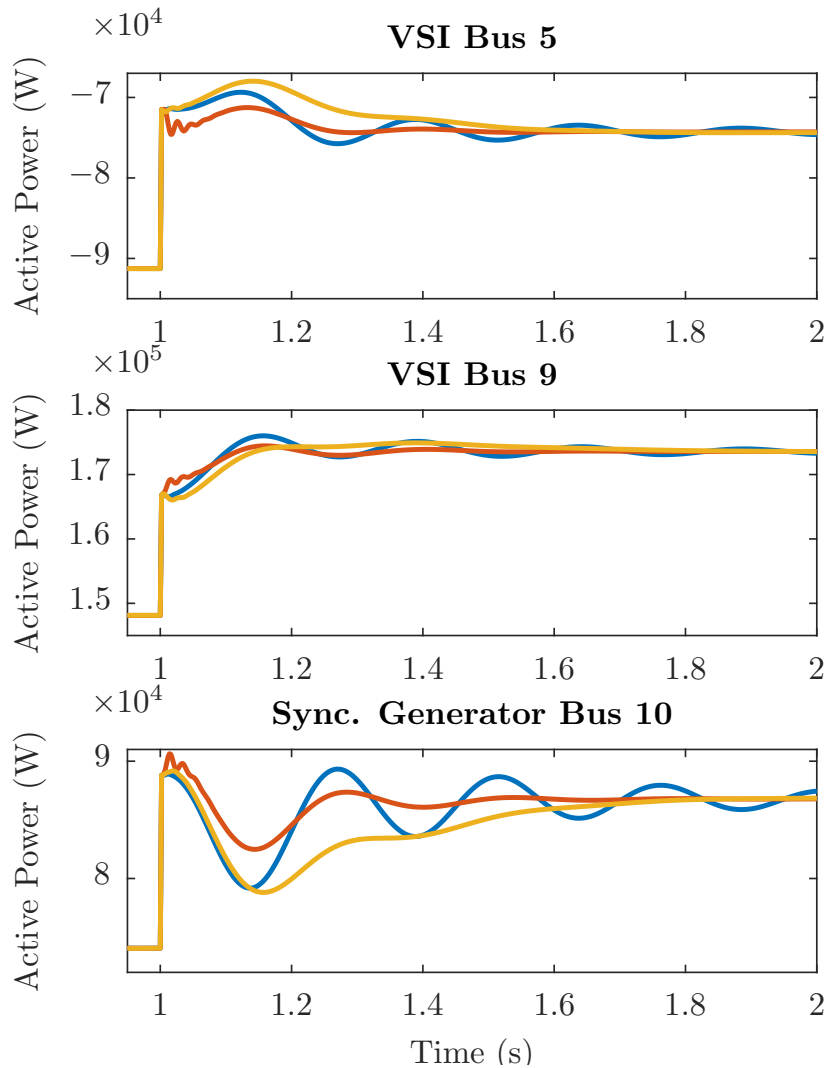

Fig. 11. Generator active output power after a load step. Blue is the droop controller, red is the improved primary controller, yellow is with the distributed controller.

realistic models of VSIs and synchronous generators, as well as other common grid components. A focus will be put on the stability of distribution grids with large amounts of photovoltaic generation and inverter-interfaced loads, which is a complex and relevant issue in todays operation of power grids.

\section{REFERENCES}

[1] J. M. Guerrero, J. Matas, L. G. de Vicuna, M. Castilla, and J. Miret, "Decentralized control for parallel operation of distributed generation inverters using resistive output impedance," IEEE Transactions on industrial electronics, vol. 54, no. 2, pp. 994-1004, 2007.

[2] Y. Du, J. M. Guerrero, L. Chang, J. Su, and M. Mao, "Modeling, analysis, and design of a frequency-droop-based virtual synchronous generator for microgrid applications," in ECCE Asia Downunder (ECCE Asia), 2013 IEEE. IEEE, 2013, pp. 643-649.

[3] Y. Hirase, O. Noro, K. Sugimoto, K. Sakimoto, Y. Shindo, and T. Ise, "Effects of suppressing frequency fluctuations by parallel operation of virtual synchronous generator in microgrids," in Energy Conversion Congress and Exposition (ECCE), 2015 IEEE. IEEE, 2015, pp. 36943701.

[4] N. Soni, S. Doolla, and M. C. Chandorkar, "Improvement of transient response in microgrids using virtual inertia," IEEE Transactions on Power Delivery, vol. 28, no. 3, pp. 1830-1838, 2013.

[5] K. De Brabandere, B. Bolsens, J. Van den Keybus, A. Woyte, J. Driesen, and R. Belmans, "A voltage and frequency droop control method for parallel inverters," IEEE Transactions on power electronics, vol. 22, no. 4, pp. 1107-1115, 2007.

[6] J. He, Y. W. Li, J. M. Guerrero, F. Blaabjerg, and J. C. Vasquez, "An islanding microgrid power sharing approach using enhanced virtual impedance control scheme," IEEE Transactions on Power Electronics, vol. 28, no. 11, pp. 5272-5282, 2013. 
[7] J. M. Guerrero, J. C. Vasquez, J. Matas, L. G. De Vicuña, and M. Castilla, "Hierarchical control of droop-controlled AC and DC microgridsa general approach toward standardization," IEEE Transactions on Industrial Electronics, vol. 58, no. 1, pp. 158-172, 2011.

[8] J. Matas, M. Castilla, L. G. de Vicuna, J. Miret, and J. C. Vasquez, "Virtual impedance loop for droop-controlled single-phase parallel inverters using a second-order general-integrator scheme," IEEE Transactions on Power Electronics, vol. 25, no. 12, pp. 2993-3002, 2010.

[9] E. Mallada, "idroop: A dynamic droop controller to decouple power grid's steady-state and dynamic performance," in Decision and Control (CDC), 2016 IEEE 55th Conference on. IEEE, 2016, pp. 4957-4964.

[10] R. Pates and E. Mallada, "Decentralized robust inverter-based control in power systems," arXiv preprint arXiv:1612.05812, 2016.

[11] J. W. Simpson-Porco, F. Dörfler, and F. Bullo, "Synchronization and power sharing for droop-controlled inverters in islanded microgrids," Automatica, vol. 49, no. 9, pp. 2603-2611, 2013.

[12] J. Schiffer, R. Ortega, A. Astolfi, J. Raisch, and T. Sezi, "Conditions for stability of droop-controlled inverter-based microgrids," Automatica, vol. 50, no. 10, pp. 2457-2469, 2014.

[13] M. H. Nazari, M. Ilic, and J. P. Lopes, "Small-signal stability and decentralized control design for electric energy systems with a large penetration of distributed generators," Control Engineering Practice, vol. 20, no. 9, pp. 823-831, 2012.

[14] A. H. Etemadi, E. J. Davison, and R. Iravani, "A decentralized robust control strategy for multi-DER microgrids - part I: Fundamental concepts," IEEE Transactions on Power Delivery, vol. 27, no. 4, pp. 1843 $1853,2012$.

[15] — - "A decentralized robust control strategy for multi-DER microgridspart II: Performance evaluation," IEEE Transactions on Power Delivery, vol. 27, no. 4, pp. 1854-1861, 2012.

[16] M. J. Hossain, M. A. Mahmud, F. Milano, S. Bacha, and A. Hably, "Design of robust distributed control for interconnected microgrids," IEEE Transactions on Smart Grid, vol. 7, no. 6, pp. 2724-2735, 2016.

[17] A. Vaccaro, G. Velotto, and A. F. Zobaa, "A decentralized and cooperative architecture for optimal voltage regulation in smart grids," IEEE Transactions on Industrial Electronics, vol. 58, no. 10, pp. 4593-4602, 2011.

[18] Q. L. Lam, A. I. Bratcu, D. Riu, and J. Mongkoltanatas, "Multi-variable H-infinity robust control applied to primary frequency regulation in microgrids with large integration of photovoltaic energy source," in Industrial Technology (ICIT), 2015 IEEE International Conference on. IEEE, 2015, pp. 2921-2928.

[19] H. Bevrani, M. R. Feizi, and S. Ataee, "Robust frequency control in an islanded microgrid: $H_{\infty}$ and $\mu$-synthesis approaches," IEEE Transactions on Smart Grid, vol. 7, no. 2, pp. 706-717, 2016.

[20] M. S. Sadabadi, A. Karimi, and H. Karimi, "Fixed-order decentralized/distributed control of islanded inverter-interfaced microgrids," Control Engineering Practice, vol. 45, pp. 174-193, 2015.

[21] M. S. Sadabadi, Q. Shafiee, and A. Karimi, "Plug-and-play voltage stabilization in inverter-interfaced microgrids via a robust control strategy," IEEE Transactions on Control Systems Technology, vol. 25, no. 3, pp. 781-791, 2017.

[22] E. H. Allen and M. Ilic, "Interaction of transmission network and load phasor dynamics in electric power systems," IEEE Transactions on Circuits and Systems I: Fundamental Theory and Applications, vol. 47, no. 11 , pp. 1613-1620, 2000.

[23] M. H. Nazari and M. Ilic, "Dynamic modelling and control of distribution energy systems: comparison with transmission power systems," IET Generation, Transmission \& Distribution, vol. 8, no. 1, pp. 26-34, 2014.

[24] X. Guo, Z. Lu, B. Wang, X. Sun, L. Wang, and J. M. Guerrero, "Dynamic phasors-based modeling and stability analysis of droopcontrolled inverters for microgrid applications," IEEE Transactions on Smart Grid, vol. 5, no. 6, pp. 2980-2987, 2014.

[25] K. D. Brabandere, "Voltage and frequency droop control in low voltage grids by distributed generators with inverter frond-end," Ph.D. dissertation, Katholieke Universiteit Leuven, FACULTEIT INGENIEURSWETENSCHAPPEN, 3001 Leuven, Belgium, 2006.

[26] C. Kammer and A. Karimi, "Robust distributed averaging frequency control of inverter-based microgrids," in Decision and Control (CDC), 2016 IEEE 55th Conference on. IEEE, 2016, pp. 4973-4978.

[27] _ "Advanced droop control in islanded microgrids using dynamic phasor models," in 20th World Congress of IFAC, Toulouse, France, 2017.

[28] V. Venkatasubramanian, H. Schattler, and J. Zaborszky, "Fast timevarying phasor analysis in the balanced three-phase large electric power system," IEEE Transactions on Automatic Control, vol. 40, no. 11, pp. $1975-1982,1995$.

[29] F. Dorfler and F. Bullo, "Kron reduction of graphs with applications to electrical networks," IEEE Transactions on Circuits and Systems I: Regular Papers, vol. 60, no. 1, pp. 150-163, 2013.

[30] P. Kundur, N. J. Balu, and M. G. Lauby, Power system stability and control. McGraw-hill New York, 1994, vol. 7.

[31] A. Karimi and C. Kammer, "A data-driven approach to robust control of multivariable systems by convex optimization," arXiv:1610.08776 [math.OC], 2016.

[32] K. Rudion, A. Orths, Z. Styczynski, and K. Strunz, "Design of benchmark of medium voltage distribution network for investigation of DG integration," 2006 IEEE Power Engineering Society General Meeting, p. 6, 2006.

[33] J. Löfberg, "YALMIP: A toolbox for modeling and optimization in MATLAB," in CACSD Conference, http://control.ee.ethz.ch/joloef/yalmip.php, 2004.

[34] MOSEK ApS, The MOSEK optimization toolbox for MATLAB manual. Version 7.1, 2015. [Online]. Available: http://docs.mosek.com/7.1/toolbox/index.html 